\title{
REFLEXIONES TEÓRICAS SOBRE LA NECESIDAD DE HUMANIZAR LAS CIENCIAS EXPERIMENTALES EN LOS PROCESOS DIDÁCTICOS
}

\section{THEORETICAL REFLECTIONS ON NEED TO HUMANIZE THE EXPERIMENTAL SCIENCES IN EDUCATIONAL PROCESSES}

\author{
ISABEL CABALLERO, ANABEl PARAMÁ Y JUAN R. COCA ${ }^{1}$ \\ juancoca@soc.uva.es \\ Universidad de Valladolid
}

\begin{abstract}
RECIBIDO: 03/12/2017
ACEPTADO: 15/02/2018
\end{abstract}

Resumen: El propósito de este artículo es evidenciar la estrecha relación existente entre las ciencias experimentales y el desarrollo de la humanidad. También se pretende mostrar el papel fundamental de su enseñanza a la hora de formar ciudadanos capaces de desenvolverse en el mundo actual y que conozcan el importante papel que la ciencia desempeña en sus vidas personales y profesionales, y, en última instancia, en nuestra sociedad.

Palabras clave: ciencia, sociedad, enseñanza, economía.

\begin{abstract}
The purpose of this article is to show the close relationship between the experimental sciences and the development of humanity. It is also intended to show the fundamental role of its teaching to train citizens to be capable to function in today's world and know the important role that science plays in their personal and professional lives, and in our society.
\end{abstract}

Keywords: science, society, education, economy.

Cuando se habla de la enseñanza de las ciencias - haremos mención exclusiva de las ciencias experimentales, aunque consideramos que también existen ciencias humanas y formales - se expone la necesidad o la dificultad de enseñar o de asumir los modelos por parte de los profesores —en el primer caso- o de educandos — en el segundo-, de cómo resolver los problemas en clase, de cómo motivar al alumno, de las diversas maneras de mejorar la enseñanza de las ciencias, etc. Pero no se suele mencionar, en demasiadas ocasiones, la relación existente entre las ciencias y la pobreza, y la docencia a este respecto. Esta carencia, se ha intentado solventar a través de los movimientos pedagógicos de CTS o de la reciente ciencia intercultural. Aun así, consideramos un poco limitados dichas corrientes, ya que se restringen a ámbitos pedagógicos muy concretos, aunque han desarrollado logros muy importantes.

\footnotetext{
${ }^{1}$ Los autores pertenecen al grupo GIR Trans-REAL lab de la Universidad de Valladolid.
} 
Por esta razón, hemos considerado que era necesario intentar cubrir este hueco y, de paso, ir poniendo las bases para el fomento de una enseñanza de las ciencias enmarcada en una concepción educativa humanista.

\section{La sociedad actual ante la ciencia}

El mundo actual se caracteriza por el avance vertiginoso de la ciencia y la tecnología, los cuales tienen un impacto directo en la sociedad. Sin embargo, a pesar de los beneficios que brindan los nuevos conocimientos a la ciudadanía, existe en esta una imagen distorsionada y alejada de la realidad tanto de la ciencia como de los científicos; se percibe erróneamente a la ciencia como inaccesible y que permite llegar a verdades absolutas e inalterables, ambos pareceres fundamentados sobre la base de la compleja terminología utilizada y de los métodos rigurosos que utiliza (Guerra Retamosa, 2004).

Diversos trabajos han puesto de manifiesto como en la sociedad existen diversas valoraciones negativas de la ciencia (Wolpert, 1992; Dunbar, 1999), En primer lugar, poderosos grupos sociales de carácter conservador y fundamentalista que no sólo la valoren negativamente sino que se oponen a la ciencia. Estos grupos, que han tenido un peso considerable en nuestro país, pueden explicar, en parte, la particular situación de la ciencia a lo largo de nuestra historia.

Pero también tenemos otros grupos que tienen una imagen negativa de la ciencia (la consideran difícil, aburrida, sólo apta para genios, etc.) y, sobre todo, de sus repercusiones peligrosas en la sociedad y el ambiente (Solbes, Montserrat, Furió, 2007). Pero tanto en la mayoría de la población como en las minorías mencionadas, se tiene una visión más negativa de las aplicaciones e influencia de la Física y Química en la sociedad y el medio que de la Biología y Geología. Respecto a las primeras se mencionan aplicaciones relacionadas con los armamentos y la energía nuclear, la contaminación, etc., y por el contrario, respecto a las segundas, se habla de la lucha contra las enfermedades, la conservación del medio, mejoras en la agricultura, etc. (Ribelles et al 1995; Solbes y Traver, 2003). Recientemente empiezan a detectarse rechazos relacionados con los organismos genéticamente modificados, la clonación, las armas bacteriológicas, etc.

Por este motivo, es necesario que la enseñanza de la ciencia no se limite a mostrar el lado positivo de esta actividad, también es imprescindible mostrar su lado negativo, que también lo tiene. A su vez, también es muy importante hacer ver que la ciencia se desarrolla, en su mayor parte, en los países desarrollados, lo 
que supone ciertas consecuencias que luego veremos. De momento, vamos a exponer donde se desarrolla la actividad científica.

\section{Actividad científica y economía}

Las actividades científicas y tecnológicas son la base del desarrollo de las sociedades, ya sea a través de su uso social o económico. Según palabras de Piñón (2005) tanto en el ámbito económico como en la política pública, el conocimiento científico es fuente crucial de valor añadido en la producción de bienes y servicios, incluso la satisfacción de las necesidades individuales y el ejercicio pleno de la ciudadanía dependen de la disponibilidad y construcciones del conocimiento científico y los productos de la acción tecnológica.

La ciencia y la tecnología actual no suelen actuar precisamente como agentes niveladores, sino que tienden más bien a hacer más ricos a los ricos y más pobres a los pobres, acentuando la desigual distribución de la riqueza entre clases sociales y naciones. Sólo una pequeña porción de la humanidad puede permitirse el lujo de un teléfono celular o de un ordenador conectado a internet.

A su vez la ciencia aplicada y la tecnología actual están en general demasiado vinculadas al efecto inmediato, al servicio de los ricos o de los gobiernos poderosos. Sólo una pequeña porción de la humanidad puede permitirse sus servicios e innovaciones Podemos preguntarnos como van a ayudarnos cosas como los aviones supersónicos, la cibernética, la televisión de alta definición o la fecundación in vitro, a resolver los grandes problemas sociales que presenta la humanidad, como comida asequible para todos, atención médica y educación accesible. Sin olvidar campos científicos tan problemáticos como la energía nuclear o la biotecnología, denunciados no solo por su aplicación militar sino también por su peligrosidad social y ambiental.

Muchos indicadores sociales muestran que, a pesar del crecimiento del comercio internacional y de la acelerada mejoría en las condiciones de vida de algunos países, la brecha entre los beneficiados y los que no lo han sido sigue en aumento. En el Informe del Programa de las Naciones Unidas para el Desarrollo (PNUD) (2005) se insiste dramáticamente en la falta de cumplimiento de las metas propuestas por los países miembros de las Naciones Unidas en los objetivos de desarrollo del Milenio. Metas encaminadas a que a fines del 2015 se viese reducida a la mitad la indigencia y a disminuir la cantidad de muertes infantiles y las enfermedades infecciosas para "liberar a nuestros semejantes de las condiciones abyectas y deshumanizadoras de la pobreza extrema" mediante la cooperación internacional dirigida a esos objetivos. Sin embargo, el mencionado 
Informe del PNUD planteaba que hasta ese momento no se habían cumplido tales objetivos y todo indicaba que, de no mediar cambios profundos en las políticas públicas de los países ricos, no se concretaría el compromiso asumido.

Este mundo dividido ha sido caracterizado claramente por Nelson Mandela, citado en el mencionado informe: "La inmensa pobreza y la obscena desigualdad son flagelos tan espantosos de esta época -en la que nos jactamos de impresionantes avances en ciencia y tecnología, industrias y acumulación de riquezas- que deben clasificarse como males sociales tan graves como la esclavitud y el apartheid" (PNUD, 2005: 4).

Para los países subdesarrollados o en vías de desarrollo el uso intensivo de la ciencia y la técnica constituye una importante estrategia para reducir la pobreza y el sufrimiento humanos. En este sentido, el Proyecto del Milenio de las Naciones Unidas, implantado en 2002 tiene entre sus ideas principales dar mayor relieve a la ciencia y la tecnología en el desarrollo, con vistas a alcanzar los Objetivos del Desarrollo del Milenio de revertir la pobreza absoluta, el hambre y la enfermedad que afectan a millones de personas (ONU, 2005).

Según los datos aportados en el Informe de la UNESCO sobre la Ciencia publicado en 2015, en la actualidad, aproximadamente 7,8 millones de científicos e ingenieros están contratados en actividades de investigación en todo el mundo. Desde 2007, el número de investigadores ha aumentado en un $21 \%$. Este notable crecimiento se refleja también en la explosión del número de publicaciones científicas.

La Unión Europea sigue siendo líder mundial en cuanto a número de investigadores, con una proporción del 22,2\%. Desde 2011, China $(19,1 \%)$ ha superado a los Estados Unidos (16,7\%). La proporción que representa el Japón a nivel mundial se ha contraído del 10,7\% (2007) al 8,5\% (2013), y la de la Federación de Rusia del 7,3\% al 5,7\%. Como se puede observar, los Cinco Grandes siguen representando el $72 \%$ de todos los investigadores, aunque sus respectivas proporciones han variado. Cabe destacar que los países de ingresos altos han cedido algo de terreno a los países de ingresos medianos altos, entre los que se incluye China; esta última representaba el $22,5 \%$ de los investigadores en 2007, pero el 28,0\% en 2013.

En el citado informe también se refleja que cuando los países están dispuestos a invertir más en personal de investigación y actividades de investigación financiadas con fondos públicos, la inclinación de las empresas a invertir en I + D también aumenta (el tamaño de los círculos). Como es obvio, la investigación financiada con fondos públicos y la financiada con fondos privados persiguen objetivos diferentes, pero su contribución al crecimiento nacional y al bienestar dependerá de hasta qué punto se complementen bien. Aunque esto es 
cierto para todos los países, con independencia de su nivel de ingresos, resulta evidente que esta correlación adquiere mayor fuerza por encima de un umbral determinado de densidad de investigadores y de intensidad de I + D financiada con fondos públicos.

Por lo que respecta a la generación del conocimiento, la Unión Europea sigue liderando el mundo de las publicaciones el 34\% del total, seguida de los Estados Unidos, con el $25 \%$. A pesar de estas impresionantes cifras, las proporciones que la Unión Europea y los Estados Unidos representan en el mundo han caído en el último quinquenio debido al meteórico ascenso de China: las publicaciones chinas prácticamente se han duplicado en los últimos cinco años hasta alcanzar el $20 \%$ del total mundial. Hace diez años, China representaba sólo el 5\% de las publicaciones mundiales. Este rápido crecimiento refleja la madurez del sistema de investigación chino, tanto en términos de inversión como de número de investigadores o publicaciones.

En este punto, debemos indicar que en los últimos cinco años puede apreciarse una tendencia hacia la convergencia, debido al descenso de inversión en el ámbito de la I + D por parte del sector público en numerosos países de altos ingresos (Australia, Canadá, los Estados Unidos, etc.) y una inversión creciente en I + D por parte de los países de ingresos más bajos. En África, por ejemplo, Etiopía ha aprovechado unas tasas de crecimiento situadas entre las más rápidas del continente para aumentar el Gasto Interior Bruto en Investigación y Desarrollo (GBID) desde el 0,24\% (2009) hasta el 0,61\% (2013) del Producto Interior Bruto (PIB). Malawi ha aumentado su propia relación GBID/PIB hasta el 1,06\%, y Uganda hasta el 0,48\% (2010), desde un nivel del 0,33\% en 2008. Tanto en África como en otras partes del mundo, se está tomando consciencia de que, para desarrollar infraestructuras modernas (hospitales, carreteras, ferrocarriles, etc.) y conseguir la diversificación económica y la industrialización, será necesaria una mayor inversión en CTI, lo que supone la constitución de una masa crítica de trabajadores cualificados.

El gasto en I + D se mueve al alza en numerosos países del África Oriental con ejes de innovación (Camerún, Kenia, Ruanda, Uganda, etc.), impulsado por un aumento de la inversión por parte de los sectores tanto público como privado.

Desde una perspectiva geográfica, la distribución de la inversión en conocimiento sigue siendo desigual. Los Estados Unidos siguen ocupando una posición predominante, con el $28 \%$ de la inversión global en I + D. China ha pasado al segundo lugar (20\%), por delante de la Unión Europea (19\%) y el Japón (10\%). El resto del mundo representa el $67 \%$ de la población mundial pero sólo el 23\% de la inversión mundial en I + D. 
El GBID incluye la inversión tanto pública como privada en I + D. La proporción del GBID ejecutada por el sector empresarial (inversión privada) tiende a ser superior en economías más centradas en un modelo de competitividad industrial basada en la tecnología, y esto se refleja en su mayor relación entre inversión privada y PIB.

Entre las economías más grandes en relación con las cuales se dispone de datos adecuados, la intensidad inversión privada/PIB sólo ha aumentado de forma apreciable en unos cuantos países, como la República de Corea y China, y en menor medida en Alemania, los Estados Unidos, Turquía y Polonia. En el mejor de los casos, se ha mantenido estable en el Japón y el Reino Unido, y retrocedido en Canadá y Sudáfrica. Teniendo en cuenta que casi uno de cada cinco seres humanos es chino, el rápido avance de la inversión privada en China ha tenido un efecto colateral de proporciones inmensas: entre 2001 y 2011, la proporción que la inversión privada combinada de China y la India representa a nivel mundial se cuadruplicó desde el $5 \%$ al $20 \%$, en gran medida en detrimento de Europa Occidental y América del Norte.

Con todos los datos expuestos, podemos afirmar que, en líneas generales, los países más empobrecidos no desarrollan ciencia. Pero esto no queda aquí. Es notorio, que a medida que pasan los años se quiere ir fomentando — cada vez más - eso que se llama I+D+i —investigación, desarrollo e innovación — que no es otra cosa que la inserción de las empresas en el mundo de la investigación científica. No vamos a entrar a valorar las bondades o maldades de este proceso, lo que queremos es intentar mostrar la situación actual. Tal y como hemos mostrado en otras ocasiones, en 1994, en Europa la financiación de la investigación científica por parte de las empresas era de casi un 53\%, en Estados Unidos era de un 59\% y en Japón algo más de un 73\%. Ahora mismo, en Estados Unidos la financiación ronda el $70 \%$ y en España, el plan nacional de $\mathrm{I}+\mathrm{D}+\mathrm{i}$ 2004-2007, habla de que la aportación de mundo empresarial a I+D+i es de algo menos del 55\%, con la intención de llegar al $60 \%$ al finalizar este plan. Todo esto, nos muestra que los gobiernos de los estados más poderosos quieren fomentar que las industrias sean las que diseñen las líneas de investigación científica. Si a esto le sumamos que la mayor parte de dicha investigación se desarrolla en esos países, podemos afirmar con rotundidad que será el sector industrial el que diseñe y coordine la líneas de investigación científica mundial.

Nadie puede pensar, que las empresas van a realizar un tipo de actividad que a corto, medio o largo plazo no les suponga beneficio. Por esta razón, podemos tener cierta seguridad de que los intereses de aquellos países con carencias económicas no serán tenidos en cuenta en dichas investigaciones ya que no tendrán dinero para costear sus propias investigaciones o para pagar los 
productos que una empresa pudiera generar. En este sentido, son clarificadoras las palabras de Invernizzi y Foladori:

\begin{abstract}
El desencuentro entre las causas reconocidas de la expansión de las enfermedades infecciosas, que son siempre socio-económicas, y las políticas de C\&T en salud parece evidente. La I\&D en salud se orienta a combatir la enfermedad, o a prevenirla en el caso de las vacunas, pero no a modificar las causas por las cuales se expanden, ya que éstas son socioeconómicas y no pueden ser asumidas por el área de la salud debido a la división social del trabajo que existe en la sociedad. Efectivamente, no puede acusarse a la OMS de, por ejemplo, no desarrollar infraestructura sanitaria o políticas de empleo. Se supone que hay otras instituciones, como por ejemplo el Banco Mundial, que se encargan del combate a la pobreza. Pero, tampoco la parte que le corresponde a las ciencias biomédicas en esa división social del trabajo parece ser solución para las grandes mayorías de la población pobre del mundo. Es bien conocido que las corporaciones farmacéuticas (farma) no investigan las enfermedades de los pobres. Según Medecins Sans Frontieres, en 2002, el $80 \%$ del mercado de fármacos estaba concentrado en Norte América, Europa y Japón, un área geográfica donde vive sólo 19\% de la población (MSF/DND, 2001). Mientras, el 90\% de la carga de enfermedad en el mundo está localizada en los países pobres, donde los enfermos no tienen la capacidad para comprar medicinas. Se estima que 18 millones de personas murieron en 2001 por enfermedades comunicables, debido a la falta de dinero para comprar medicinas o porque no hay medicinas apropiadas para determinadas enfermedades" (Invernizzi y Foladori, p. 141 y sig.).
\end{abstract}

Esto nos muestra la situación del mundo, en la que la pobreza es el factor determinante, tanto para el ser humano. Pero, además, nos damos cuenta que la ciencia se ha convertido en uno de los factores más importantes en el mantenimiento de las diferencias entre los países desarrollados y países en vía de desarrollo, ya que serán los primeros los que tienen más posibilidades de configurar sus criterios científicos como fundamentación de lo que debe ser investigado (Coca, 2004a). Esto sucede, sobre todo, porque esta actividad constituye uno de los principales estandartes de la sociedad de consumo y porque relega, todavía más, a aquellas sociedades no occidentales. Por esta razón, los países y los científicos de todo el mundo, tienen que tener clara la necesidad de un marco ético para que los posibles riesgos desarrollados por la ciencia no den lugar a procesos escatológicos (Coca, 2006a). Además, todas las culturas deben ser tenidas en cuenta tanto a la hora de desarrollar la actividad científica como en los objetivos buscados en dicha actividad: ciencia intercultural (Coca, 2004b y 2005a). Por último, no podemos olvidarnos del posible papel que la ciencia puede tener a la hora de desarrollar un mundo más equitativo, próspero y sostenible. Para ello, hay que revisar las políticas científicas (Coca, 2005b) y 
favorecer una distribución equitativa de los beneficios aportados por esta actividad.

\section{La enseñanza de las ciencias como agente nivelador de las diferencias económicas}

Con relativa frecuencia, determinadas personas, se sienten incapaces de controlar ciertos productos tecnológicos o de afrontar simples razonamientos relacionados con la ciencia. La educación debería disminuir esa inseguridad, que algunos ciudadanos tienen, de tal forma que se pudiera disfrutar de los crecientes beneficios de la era de la ciencia y la tecnología, garantizando al mismo tiempo, la protección de la salud y el medio y contribuyendo con el conocimiento a la toma de decisiones sobre el desarrollo científico y tecnológico en el que estamos inmersos, y sus consecuencias. Por tanto la alfabetización científica será necesaria para contribuir a formar ciudadanos, y en su caso futuros científicos, que sepan desenvolverse en un mundo como el actual y que conozcan el importante papel que la ciencia desempeña en sus vidas personales y profesionales, y en nuestra sociedades. Ciudadanos cuya formación les permita reflexionar y tomar decisiones apropiadas en temas relacionados con la ciencia y la tecnología (Aikenhead, 1985; Bingle y Gaskell, 1994; Gil et al., 1991; Solbes y Vilches, 1997).

Así mismo se considera conveniente presentar las aportaciones a la ciencia realizadas en países que no son grandes potencias científicas y los obstáculos que se han planteado al desarrollo de la ciencia en dichos países a lo largo de la historia.

Desde esta perspectiva se pretende promover que los estudiantes lleguen a ser capaces de realizar evaluaciones sobre diversos desarrollos científicos y tecnológicos, en particular, la evaluación de riesgos y de impacto social y ambiental. Esto debe conducir a valoraciones, a juicios éticos que pueden realizarse atendiendo a la contribución de dichos desarrollos a la satisfacción de necesidades humanas (sin olvidar que el conocimiento es una de ellas) y a la solución de los problemas del mundo.

Se quiere, así mismo, poner de manifiesto los intereses y valores subyacentes en las opciones y decisiones sobre ciencia y tecnología de diversos actores sociales (el estado, las empresas transnacionales, científicos, empresarios, etc.), ocultos por la aparente neutralidad de la ciencia y tecnología. Se podrá ver así que existen diferentes valoraciones y que es necesario compararlas, argumentarlas, para llegar a conclusiones y traducir los argumentos en implicación en políticas 
públicas (Hodson, 1994): escritos y declaraciones, solicitudes, votaciones, participación en proyectos... sin olvidar el importante y creciente papel de las Organizaciones no Gubernamentales $u$ otras organizaciones sociales en el denominado entramado sociotecnológico.

De esta forma, se contribuirá a combatir la imagen pública negativa de la ciencia que hemos analizado anteriormente. Para ello, se debe plantear claramente la contribución de la ciencia al desarrollo general de la humanidad y a una concepción del mundo basada en la racionalidad y el espíritu crítico frente a cualquier tipo de fundamentalismo (especialmente aquéllos que han pretendido tener un origen científico, como el darwinismo social, la eugenesia, el racismo, etc.) y pseudocientifismo (como la astrología, la ufología, etc.).

También será necesario presentar ejemplos de responsabilidad social de científicos y técnicos. Por ejemplo, la situación que llevó a Einstein, Born, Pauling, etc., a denunciar cómo el uso irracional de la ciencia en la guerra fría ponía en peligro la paz entre las naciones o, en la actualidad, la de tantos otros cuyas investigaciones han puesto de manifiesto, pese a la oposición de muchas empresas, que la radiactividad es perjudicial, el tabaco y otros productos son cancerígenos, los CFCs destruyen la capa de ozono, etc. (Sánchez Ron, 1994).

Todo esto debe llevar a una valoración más matizada de cada una de las investigaciones y desarrollos de la ciencia y la tecnología.

Si nos centramos en nuestro país, debemos decir que la enseñanza de las ciencias, muestra graves carencias. Ello es debido a que, se transmite una imagen de la ciencia descontextualizada, donde no se tienen en cuenta aspectos cualitativos de tipo histórico, social o cultural. No obstante, Vilches nos advierte que recientemente se ha puesto de manifiesto que enseñar una ciencia más humana, estimular el estudio de la ciencia como vehículo cultural, conectar la ciencia con los problemas reales del mundo, son un telos necesario en la docencia (Vilches, 2002).

Ello conlleva - nos sigue diciendo - mostrar una imagen más contextualizada del conocimiento científico, lo que permite identificar los problemas reales de la vida real. Por otro lado, es necesario profundizar en la propia construcción del conocimiento científico, lo que mejorará nuestra comprensión de esta actividad. Además, es importante - a la hora de enseñar - comprometer a los jóvenes en la solución de los graves problemas que hipotecan el futuro de la humanidad. Por último, la enseñanza de las ciencias tiene que transformarse en un elemento fundamental de la cultura, para la formación de personas responsables, que puedan participar activamente en los asuntos sociales. 
Todo lo dicho, nos permite afirmar que la relación entre la ciencia y la sociedad, no puede quedarse en una lección, en unas cuantas clases, etc. Debe ser un tipo de orientación educativa, a todos los niveles, de tal manera que cualquier aspecto que se explicase, fuese relacionado estrechamente con algún ámbito social.

\section{Reflexiones finales}

Además de la estrecha relación entre ciencia y sociedad, ampliamente analizada a lo largo de todo este texto, no queremos acabar este trabajo sin analizar otro concepto directamente relacionados con los anteriores, el concepto de interculturalidad.

Cuando hablamos de ciencia intercultural —en el ámbito educativo-, estamos mencionando tres tipos de bloques de trabajo diferentes (Cabo y Enrique, 2004). En primer lugar, aquellos trabajos que muestran ésta como una alternativa a las concepciones tradicionalistas de la actividad científica. En segundo lugar estarían aquellos trabajos que aportan base teórica y empírica sobre cómo influye la cultura sobre nuestra actividad. Y, en último lugar, tendríamos los trabajos de ciencia y género. Esta nueva concepción de la ciencia, consiste en lo siguiente (Cabo y Enrique, 2004):

a. Implica considerar que la ciencia puede ser distinta al positivismo, en el sentido de una ciencia tentativa o ciencia posnormal.

b. Conlleva la aceptación de la relación entre la cultura y la ciencia, dicho de otro modo, entender la ciencia como práctica sociocultural.

c. Supone entender la ciencia de un modo más constructivista, dejando a un lado las posturas más individualistas y cognitivas.

d. A nivel didáctico, plantea la convivencia de una ciencia personal, con una ciencia escolar y occidental; y la relación de cada una de ellas con su propio escenario.

e. Implica, a nivel curricular, centrarse más que el sujeto que aprende, en el escenario sociocultural del que aprende.

f. Supone la defensa de los valores de justicia social, solidaridad, tolerancia, igualdad de oportunidades a favor de los más necesitados y ser conscientes de la politización del currículum.

Cabo y Enrique añaden un punto más, la consideración de que todo ello encaja en una orientación CTS. En nuestro caso, y para evitar posibles confusiones, no consideramos que una encaje en la otra; ya que puede parecer que la concepción de ciencia intercultural debe estar dentro de una consideración CTS.

Nosotros afirmaremos que la ciencia intercultural, es una manera de entender esta actividad equiparable al movimiento CTS. La diferencia se encuentra en el 
hecho de que la ciencia intercultural es un movimiento — valga la redundancianetamente científico, mientras que el movimiento CTS es fundamentalmente social. Por tanto, entendemos por ciencia intercultural al conjunto de cambios y reformas curriculares que se deben realizar en el contexto educativo de las ciencias experimentales para responder a la existencia de un contexto multicultural. Por esta razón, creemos que se complementan muy bien, pero ninguno de ellos está dentro del otro.

\section{Bibliografía}

Aikenhead, G.S. (1985). Collective decision making in the social context of science. Science Education, 69 (4), 453-475.

Bingle, W. y Gaskell, P.J. (1994). Scientific Literacy for decision making and the social construction of scientific knowledge. Science Education, 78(2), 185-201.

Cabo Hernández, J. M. y Enrique Mirón, C. (2004): "Hacia un concepto de ciencia intercultural", Enseñanza de las ciencias, 22 (1), 137-146.

Coca, J. R. (2004a): “Ciencia y pobreza", Acontecimiento, 73 (4), 26-28.

- (2004b): "Hacia una ciencia personalista y comunitaria", Analogía filosófica, XVIII, (2), 45-56.

- (2005a): "Una propuesta de cambio de las políticas científicas", en Actas I

Congreso internacional de personalismo comunitario: Democracia, persona y participación social, Madrid: Emmanuel Mounier, 157-161.

— (2005b): "El progreso de la actividad científica", Analogía filosófica, XIX (1), 13-43.

- (2006a): "Los nuevos riesgos de la nueva genética", Acontecimiento, $\mathrm{n}^{\circ} 81$, vol. 4, 25-26.

- (2006b): "Unha proposta personalista e comunitaria sobre a pedagoxía da actividade científica", Boletín das Ciencias, diciembre, (aceptado).

Dunbar, R. (1999). El miedo a la ciencia. Alianza, Madrid.

Gil, D. Carrascosa, J., Furió, C. y Martínez y Torregrosa, J. (1991). La enseñanza de las ciencias en la educación secundaria. Barcelona: Horsori.

Guerra Retamosa, C. (2004). Laboratorios y batas blancas en el cine. Revista Eureka sobre Enseñanza y Divulgación de las Ciencias. 1 (1): 52-63.

Hodson, D. (1994). Hacia un enfoque más crítico del trabajo de laboratorio. Enseñanza de las Ciencias, 12(3), 299-313.

Invernizzi, N. y Foladori, G. (2005): "Ciencia y desarrollo en los países pobres: Reflexiones sobre la investigación y desarrollo en salud", Analogía filosófica, XIX (1), 139-169. 
Jordi Solbes, Rosa Montserrat y Carles Furió. (2007). El desinterés del alumnado hacia el aprendizaje de la ciencia: implicaciones en su enseñanza. Didáctica de las Ciencias Experimentales y Sociales, 21, 91-117

Organización de las Naciones Unidas para la Educación, la Ciencia y la Cultura, (2015). Informe de la UNESCO sobre la Ciencia. Hacia 2030. UNESCO. Francia.

Programa de las Naciones Unidas para el Desarrollo (PNUD) (2005): Informe sobre Desarrollo Humano 2005. Disponible en hdr.undp.org/en/media/HDR05_sp_complete.pdf.

Pintos, J. L. (2002): "Educación, artes e novas posibilidades", Revista Galega do Ensino, n $^{\circ} 36$ (Outubro), 23-45.

Piñón, F. (2005). Ciencia y tecnología en América Latina: una posibilidad para el desarrollo, recuperado el 20 de Enero de 2016 http://www.oei.es/salactsi/pinon.pdf.

Sánchez Ron, J.M. (1994): ¿El conocimiento científico prenda de felicidad? En Nadal, J. (Ed.), El mundo que viene, Madrid, Alianza.

Solbes, J. (2011). ¿Por qué disminuye el alumnado de ciencias? Alambique, 67, 53-62.

Solbes, J. y Traver, M.J. (1996). La utilización de las historia de las ciencias en la enseñanza de la Física y la química. Enseñanza de las ciencias, 14 (1), 103-112.

Solbes, J. y Vilches, A. (1997) STS interactions and the teaching of Physics and Chemistry. Science Education, 81, 4, 337-386.

Vilches, A. (2002): "La introducción de las interacciones ciencia, técnica y sociedad (CTS). Una propuesta necesaria en la enseñanza de las ciencias", en: VV.AA. Las ciencias en la escuela. Teorías y prácticas, Barcelona, Graó/Laboratorio Educativo, 37-48.

Wolpert, L. (1992). The Unnatural Nature of Science. London: Faber \& Faber. Traducción de A. Linares (1994): La naturaleza no natural de la ciencia. Madrid: Acento Editorial. 